\title{
Aspergillus fumigatus sensitization among asthma COPD overlap patients
}

The Egyptian Journal of Immunology Volume 28 (3), 2021: 85-93 www.Ejimmunology.org

\author{
Mohamed A Elshayeb ${ }^{1}$, Noha 0 Ahmed ${ }^{2}$, Nancy S \\ Wahba $^{3}$, Mahetab H Elsaeed ${ }^{1}$, Asmaa M Abd \\ ElGwad $^{4}$ and Sylvia T Kamal ${ }^{1}$
}

\author{
${ }^{1}$ Internal Medicine, Allergy \& Clinical Immunology \\ Department, Faculty of Medicine, Ain shams University, \\ Cairo, Egypt. \\ ${ }^{2}$ Pulmonology Department, Faculty Of Medicine, Ain Shams \\ University, Cairo, Egypt. \\ ${ }^{3}$ Clinical Pathology Department, Faculty of Medicine, Ain \\ Shams University, Cairo, Egypt.
}

\begin{abstract}
${ }^{4}$ Department of Medical biochemistry \& molecular biology, Faculty of Medicine, Ain shams University, Cairo, Egypt.

Corresponding author: Sylvia T. Kamal, Internal Medicine, Department of Allergy \& Clinical Immunology, Ain Shams University, Cairo, Egypt Email: drsylviatalaat@gmail.com.
\end{abstract}

\begin{abstract}
Recently, many researchers are interested in studying asthma COPD overlap (ACO) group features. Sensitization to Aspergillus has been linked to increased severity of asthma; however, limited data is available about fungal sensitization in ACO group. This study was designed to determine the prevalence of Aspergillus fumigatus (A. fumigatus) sensitization among ACO patients in comparison to asthma patients. This cross-sectional study included 30 patients with $A C O$, and compared them to 30 asthmatic patients, as regards sensitization to $A$. fumigatus. Sensitization was diagnosed using skin prick test and specific IgE. Blood eosinophil count, total IgE and pulmonary functions results were also recorded. Thirteen patients with ACO (43.3\%) were sensitized to A. fumigatus compared to 8 asthmatic cases (26.7\%), $P$ value 0.17 . Blood eosinophil count and total IgE were significantly higher among ACO patients compared to asthma group ( $P$ values $0.003,0.007$ respectively). Blood eosinophil count was significantly higher among $A$. fumigatus sensitized ACO patients ( $400 \mathrm{cells} / \mu \mathrm{L})$ compared to 320 cells/ $\mu \mathrm{L}$ in non-sensitized ACO subgroup ( $P$ value 0.01 ). Otherwise, they were comparable regarding pulmonary functions and total IgE. In conclusion, Sensitization to A. fumigatus is higher among patients with ACO than that in patients with asthma but without a statistical significance. Moreover, Sensitized ACO patients showed significantly higher blood eosinophil count than non-sensitized ones.
\end{abstract}

Keywords: Asthma COPD overlap, asthma, Aspergillus fumigatus, asthma

Date received: 25 March 2021; accepted: 28 May 2021

\section{Introduction}

Obstructive lung diseases comprise both asthma and chronic obstructive pulmonary disease (COPD). This group is considered a global health problem, which consumes healthcare resources. Although both are chronic inflammatory diseases, yet they have different underlying pathogenesis; hence different response to antiinflammatory medications. ${ }^{1}$ The predominant cells in asthma pathway are eosinophils, mast cells, and T helper2 cells; whereas those in COPD are neutrophils, macrophages, and CD8 ${ }^{+}$ T-lymphocytes ${ }^{2}$. Variation of symptoms (both in intensity and over time) and Variable expiratory airflow limitation can distinguish asthma from 
COPD. COPD is characterized by persistent respiratory symptoms and airflow limitation due to marked exposure to noxious particles or gases. $^{3}$

Patients with clinical features of both asthma and COPD are often observed in clinical practice. This remark has led to the introduction of the term "asthma-COPD overlap" (ACO). Although many studies were interested in studying ACO in the last decade; however so far, no definite criteria are widely agreed upon. The main proposed criteria for diagnosis include being older than 40 years old, persistent airflow limitation, and history of asthma or evidence of partial bronchodilator reversibility. ${ }^{4,5}$

The prevalence of ACO ranges from $0.3 \%$ to $5 \%$ in the general population. It represents $3.2 \%$ to $51.4 \%$ among patients with asthma and $12.6 \%$ to $55.7 \%$ among those with COPD. ${ }^{1}$ Studies revealed that patients with ACO have poorer outcomes than those with either asthma or COPD only. ${ }^{6}$ Unfortunately, treatment specifically targeted at this phenotype is noticeably lacking because patients with ACO are often precluded in clinical trials of novel medications for asthma and COPD. As a result, diagnosis and management of these patients poses a problem for most physicians. ${ }^{4}$

Another pivotal factor in management of asthma patients is sensitization to fungi, which was linked to severe asthma. ${ }^{7}$ Fungal sensitization is diagnosed either by skin prick test to fungal antigen(s) or an increase in specific IgE antibodies. ${ }^{8,9}$ Aspergillus species, especially Aspergillus fumigatus (A. fumigatus), are incriminated as one of the strongest fungal genera which worsen asthma and COPD control and increase risk of bronchiectasis as well. ${ }^{10,11}$

In the last decade, there is a growing body of literature that recognizes features of ACO; nevertheless, there has been limited data about fungal sensitization among those patients. This study attempts to highlight the prevalence of $A$. fumigatus sensitization among ACO patients and compare it to asthmatic patients. Identifying the magnitude of fungal sensitization among the ACO group may provide new insights into the management of those patients.

\section{Patients and Methods}

\section{Study design and participants}

This cross-sectional study encompassed 30 patients with bronchial asthma (diagnosed according to the Global Initiative for Asthma ${ }^{12}$ exceeding 40 years of age, and 30 patients with ACO (diagnosed according to criteria suggested by Sin et al. $)^{4}$. Diagnosis of ACO was made when the patient fulfilled all the major criteria (postbronchodilator $\mathrm{FEV}_{1} / \mathrm{FVC}$ ratio less than 0.7 in subjects exceeding 40 years of age, tobacco smoking at least 10 pack-years or equivalent air pollution. Patient should be previously diagnosed as asthma or achieving a bronchodilator response (BDR) of forced expiratory volume in one second $\left(\mathrm{FEV}_{1}\right)$ more than $400 \mathrm{ml}$. Additionally, at least one minor criterion was met (peripheral blood eosinophil count more than 300 cells/ $\mu \mathrm{L}$ or history of atopy, or BDR of FEV1 more than $200 \mathrm{ml}$ and $12 \%$ from baseline values). All patients were recruited from the allergy and immunology and chest outpatient clinics of Ain Shams University hospitals, in the period between 2018 and 2019. Both groups were comparable regarding age and sex.

After approval of the study protocol by the Ethical Committee of the Ain Shams University, a written informed consent was obtained from each participant. Exclusion criteria of this study comprised patients during exacerbation, immunocompromised patients and those having any other pulmonary comorbidities. Also, patients with restrictive pattern at pulmonary functions, or on chronic corticosteroid therapy were not allowed in this research.

Demographic characteristics including age, gender, smoking status and exposure to pollution were collated. Selected patients underwent comprehensive clinical history taking (including disease duration, drug history and the presence of other atopic diseases) and thorough physical examination.

\section{Pulmonary Function Test}

Pulmonary functions were measured Pre and Post-bronchodilator (10-15 min after 200-400 albuterol or equivalent) using a standardized equipment (SpiroMaster PC-10. Manufactured by: CHEST M.I., INC.), according to the American Thoracic Society/European Respiratory Society 
guidelines. The post-bronchodilator $\mathrm{FEV}_{1}$, forced vital capacity (FVC) and the ratio between them (post bronchodilator $\mathrm{FEV}_{1} / \mathrm{FVC}$ ratio) were obtained. ${ }^{13} \mathrm{~A}$ low ratio indicates an obstructive defect.

Sample collection, preparation, and analytical methods

-Laboratory investigations included the following: a) Complete blood count ( $\mathrm{CBC}$ ) and absolute eosinophil count. b) Assay of serum total IgE c) Assay of specific IgE (slgE) for $A$. fumigatus.

-A total volume of $5 \mathrm{~mL}$ venous blood sample was drawn from all subjects included in the study using aseptic venipuncture technique: $2 \mathrm{~mL}$ were collected in vacutainer EDTA tubes for $C B C$ and eosinophil count, and $3 \mathrm{~mL}$ in plain tubes for the determination of serum total IgE and slgE for Aspergillus fumigatus. The serum was separated by centrifugation and kept frozen at -20c.

a) The CBC and blood absolute eosinophil count were done using a Coulter Counter (Beckman Coulter, California 92821, USA). The reference range for eosinophils in blood (percentage) is $0.0-6.0 \%$, while the reference range for absolute count of blood eosinophils is 30-350/ $\mu \mathrm{L}$.

b) Total serum IgE was determined using a commercially available ELISA kit supplied by BIOS Microwell Diagnostic Systems (Beijing, China) following the manufacturer's instructions. The absorption value for each sample was used to determine the proper IgE concentration from a standard curve. According to the manufacturer's directions, total IgE levels more than $100 \mathrm{IU} / \mathrm{mL}$ were considered abnormal.

c) Assay for serum A. fumigatus,, slgE was performed using a commercial ELISA kit provided by RIDASCREEN ${ }^{\circledR}, R$-Biopharm AG (64297 Darmstadt, Germany). The color intensity was directly proportional to the level of slgE antibodies of $A$. Fumigatus present in the serum. Photometric measurement was carried out and a standard curve was set up and patients' samples were calculated. According to manufacturer's recommendation, the test was considered positive at $>0.35 \mathrm{kU} / \mathrm{L}$.

\section{Allergy skin test}

Skin prick test was done for all patients with standardized commercial extracts of $A$. fumigatus provided by Omega Laboratory (Montreal, Canada). Histamine and saline were used as positive and negative controls respectively. A skin test was considered valid if the difference in mean wheal diameters between the positive and negative controls was at least $1 \mathrm{~mm}$. A positive response was defined as a wheal diameter of $3 \mathrm{~mm}$ greater than the negative control, 20 minutes after application. ${ }^{14}$

Statistical data analysis:

Data were analyzed using IBM SPSS software package version 20.0. (Armonk, NY: IBM Corp). Qualitative data were illustrated in the form of number and percentage. Parametric quantitative data were described using mean and standard deviation (SD), while nonparametric was in the form of median and interquartile range (IQR). A $p$-value of less than 0.05 was considered significant. Chi-square test was used to compare categorical data, while Independent $\mathrm{t}$ test and Mann Whitney were used to compare continuous data normally and non-normally distributed respectively.

\section{Results}

In this study, 30 patients with ACO were compared to 30 patients with asthma. No significant difference was evident between both groups regarding age, sex and disease duration. Median disease duration among patients with ACO was 15 versus 10 years among patients with asthma $(P=0.066)$. All ACO patients were smokers, where $43.3 \%$ of them were active smokers with median smoking index 25 packs/year. The rest were passive smokers for more than 20 years. Asthmatic group encompassed only 2 smokers (1 active and 1 passive). No significant difference was evident between both groups regarding the presence of other atopic diseases (associated allergic rhinitis and/or urticaria) ( $P$ value 0.592). As for laboratory investigations, blood eosinophil count and total IgE were significantly higher among ACO patients compared to asthma group (350 vs 170 cells/ $\mu \mathrm{L}, 180$ vs $95 \mathrm{IU} / \mathrm{ml}$; $P$ values $0.003, \quad 0.007$ respectively). Concerning spirometry results, ACO patients showed significantly lower post bronchodilator FEV1\% of 
predicted than that of asthma, where $P<0.001$ $(65.20 \pm 9.94$ compared to $84.28 \pm 7.81$ respectively). Likewise, post bronchodilator FEV1/FVC\% was significantly lower among ACO patients compared to patients with asthma $(61.93 \pm 7.59$ vs $77.50 \pm 9.30, P<0.001)$. (Table 1)

Table 1. Comparison between ACO and asthma patients regarding demographic data, laboratory results and pulmonary function tests:

\begin{tabular}{|c|c|c|c|}
\hline & $\begin{array}{c}\text { ACO } \\
n=30\end{array}$ & $\begin{array}{c}\text { Asthma } \\
n=30\end{array}$ & $P$ value \\
\hline \multicolumn{4}{|l|}{ Sex: } \\
\hline Male & $15(50 \%)$ & $8(26.7 \%)$ & \multirow{2}{*}{ NS } \\
\hline female & $15(50 \%)$ & $22(73.3 \%)$ & \\
\hline Age (years) & $48.77 \pm 6.58$ & $47.63 \pm 7.42$ & $N S^{b}$ \\
\hline Disease duration (years) & $15(10-20)$ & $10(6-17.75)$ & $\mathrm{NS}^{\mathrm{c}}$ \\
\hline \multicolumn{4}{|l|}{ Smoking: } \\
\hline Active smokers (\%) & $13(43.3 \%)$ & $1(3.3 \%)$ & \multirow{3}{*}{$<0.001^{\mathrm{a}}$} \\
\hline Passive smokers (\%) & $17(56.7 \%)$ & $1(3.3 \%)$ & \\
\hline Nonsmokers (\%) & $0(0 \%)$ & $28(93.3 \%)$ & \\
\hline Associated atopic disease: & $20(66.7 \%)$ & $18(60 \%)$ & $N S^{a}$ \\
\hline Total IgE (IU/ml) & $180(74.75-349.75)$ & $95(24-150.50)$ & $0.007 c$ \\
\hline Blood Eosinophils (cells/ $\mu \mathrm{L}$ ) & $350(300-585)$ & $170(107.50-392.50)$ & $0.003^{c}$ \\
\hline FEV1 \% pred (After BD) & $65.20 \pm 9.94$ & $84.28 \pm 7.81$ & $<0.001^{b}$ \\
\hline FEV1/FVC\% (After BD) & $61.93 \pm 7.59$ & $77.50 \pm 9.30$ & $<0.001^{b}$ \\
\hline
\end{tabular}

a: Chi- square test, b: Independent t test, c: Mann Whitney test, categorical data are expressed as $\mathrm{n}$ (\%) numerical, mean \pm SD or median (interquartile range), ACO: asthma COPD overlap, BD: bronchodilator, FEV1\%: forced expiratory volume in first second, FEV1/FVC\%: forced expiratory volume in first second: forced vital capacity \% pred: percentage of predicted. $P>0.05$ is not significant (NS).

Table 2 illustrates the results of skin prick test and specific IgE in both groups. Sensitization to A. fumigatus was higher among patients with ACO 13 (43.3\%) (by slgE and/or skin test) compared to $8(26.7 \%)$ of asthma patients; however, this was not significant statistically ( $p$ value 0.17). Specific IgE titers were comparable in both groups ( $P$ value 0.25 ). Concordance between skin test and slgE test results in both groups was $85.7 \%$.

Table 2. Comparison between ACO and asthma regarding $A$. fumigatus sensitization:

\begin{tabular}{lccc}
\hline & ACO $(\mathrm{n}=30)$ & Asthma $(\mathrm{n}=30)$ & $P$ value \\
\hline SPT for A. fumigatus & $13(43.3 \%)$ & $7(23.3 \%)$ & $\mathrm{NS}^{\mathrm{a}}$ \\
\hline slgE for A. fumigatus & $12(40.0 \%)$ & $7(23.3 \%)$ & $\mathrm{NS}^{\mathrm{a}}$ \\
\hline Both SPT and slgE & $12(40.0 \%)$ & $6(20.0 \%)$ & $\mathrm{NS}^{\mathrm{a}}$ \\
\hline Total positive A. fumigatus & $13(43.3 \%)$ & $8(26.7 \%)$ & $\mathrm{NS}^{\mathrm{a}}$ \\
\hline slgE titre (median ) & $0.23(0.17-0.53)$ & $0.23(0.14-0.33)$ & $\mathrm{NS}^{\mathrm{b}}$ \\
\hline
\end{tabular}

a: Chi- square test, b: Mann Whitney test, categorical data are expressed as $\mathrm{n}(\%)$ numerical, or as median (interquartile range), ACO: asthma COPD overlap, A. fumigatus: Aspergillus fumigatus, SPT: skin prick test, slgE: specific immunoglobulin E. $P>0.05$ is not significant (NS).

Surprisingly, on comparing $A$. fumigatus sensitized with non-sensitized ACO patients in different study parameters, only median blood eosinophil count was significantly higher among sensitized ACO patients (400 vs 320 cells/ $\mu \mathrm{L}, P=$ 0.017). Otherwise, they were comparable regarding pulmonary functions, total IgE and smoking status. On the other hand, sensitized 
asthmatic patients showed significantly lower post bronchodilator $\mathrm{FEV}_{1} \%$ and $\mathrm{FEV}_{1} / \mathrm{FVC}$ ratio ( $P=0.001,0.002$ respectively) compared to non-sensitized asthma patients. Otherwise other parameters were comparable. (Table 3)

Table 3. Comparison between sensitized and non-sensitized ACO and asthma patients:

\begin{tabular}{|c|c|c|c|c|c|c|}
\hline & $\mathrm{ACC}$ & & & Ast & hma & \\
\hline & $\begin{array}{l}\text { Positive } A \text {. } \\
\text { fumigatus } \\
\quad(n=13)\end{array}$ & $\begin{array}{l}\text { Negative } \\
\text { A. fumigatus } \\
(\mathrm{n}=17)\end{array}$ & $P$ & $\begin{array}{l}\text { Positive } \\
\text { A. fumigatus } \\
(\mathrm{n}=8)\end{array}$ & $\begin{array}{c}\text { Negative } \\
\text { A. fumigatus } \\
\text { (n-22) }\end{array}$ & $\begin{array}{c}P \\
\text { value }\end{array}$ \\
\hline Smoking & & & & & & \\
\hline Active & $5(38.5 \%)$ & $8(47.1 \%)$ & $\mathrm{NC}^{\mathrm{a}}$ & $0(0 \%)$ & $1(4.5 \%)$ & $\mathrm{NC}^{\mathrm{a}}$ \\
\hline Passive & $8(61.5 \%)$ & 9 (52.9\%) & NS & $0(0 \%)$ & $1(4.5 \%)$ & NSS \\
\hline Non smoker & $0(0 \%)$ & $0(0 \%)$ & & $8(100 \%)$ & 20 (90.9\%) & \\
\hline Age (years) & $46.54 \pm 4.67$ & $50.47 \pm 7.42$ & $N S^{b}$ & $47.63 \pm 5.53$ & $47.64 \pm 8.11$ & $N S^{b}$ \\
\hline $\begin{array}{l}\text { Disease duration } \\
\text { (years) }\end{array}$ & $17(10-22.50)$ & $15(11-18)$ & $N S^{c}$ & $6(5.25-15.25)$ & $12(8-20.00)$ & $N S^{c}$ \\
\hline $\begin{array}{l}\text { Total IgE level } \\
\text { (IU/ml) }\end{array}$ & $250(92.5-1100)$ & $170(62-272.5)$ & $N S^{c}$ & $101(21-716)$ & $\begin{array}{c}87.5(24- \\
129.75)\end{array}$ & $N S^{c}$ \\
\hline $\begin{array}{l}\text { Eosinophils } \\
\text { (cells } / \mu \mathrm{L} \text { ) }\end{array}$ & $400(350-790)$ & $320(220-390)$ & $0.017^{c}$ & $\begin{array}{c}355(166.75- \\
421)\end{array}$ & $160(87.5-332)$ & $N S^{c}$ \\
\hline $\begin{array}{l}\text { FEV1\% pred (after } \\
\text { BD) }\end{array}$ & $67.85 \pm 6.49$ & $63.18 \pm 11.73$ & $N S^{b}$ & $77.19 \pm 8.43$ & $86.86 \pm 5.87$ & $0.001^{b}$ \\
\hline $\begin{array}{l}\text { FEV1/FVC (after } \\
\text { BD) }\end{array}$ & $63.91 \pm 5.67$ & $60.42 \pm 8.65$ & $N S^{b}$ & $69.16 \pm 7.39$ & $80.53 \pm 8.07$ & $0.002^{b}$ \\
\hline
\end{tabular}

Table 4 provides a comparison between all $A$. fumigatus sensitized patients in the study $(n=21)$ to non- sensitized $(n=39)$. Blood eosinophil count was significantly higher among sensitized patients $(P=0.002)$. Moreover, post- bronchodilator $\mathrm{FEV}_{1} / \mathrm{FVC}$ was significantly lower in sensitized patients $(P=0.025)$. Apart from that, they were comparable regarding age, disease duration, total IgE and FEV1\%.

Table 4. Comparison of clinical and laboratory findings between A. fumigatus sensitized and nonsensitized patients.

\begin{tabular}{lccc} 
& $\begin{array}{c}\text { A. fumigatus sensitized } \\
(\mathrm{n}=21)\end{array}$ & $\begin{array}{c}\text { A. fumigatus Non-sensitized } \\
(\mathrm{n}=39)\end{array}$ & $P$ value \\
\hline Age (years) & $46.95 \pm 4.90$ & $48.87 \pm 7.84$ & $\mathrm{NS}^{\mathrm{a}}$ \\
\hline ACO $(\mathrm{n}=30)$ & $13(62 \%)$ & $17(43.5 \%)$ & $\mathrm{NS}^{\mathrm{c}}$ \\
\hline Disease duration (years) & $14(6-20)$ & $14(9-20)$ & $\mathrm{NS}^{\mathrm{b}}$ \\
\hline Total IgE level $(\mathrm{IU} / \mathrm{ml})$ & $170(72.5-1000)$ & $110(28.9-225)$ & $\mathrm{NS}^{\mathrm{b}}$ \\
\hline Eosinophils (cells/ $\mu \mathrm{L})$ & $400(335-665)$ & $250(110-350)$ & $0.002^{\mathrm{b}_{\mathrm{b}}}$ \\
\hline FEV1\% pred (after BD) & $71.40 \pm 8.47$ & $76.54 \pm 14.78$ & $\mathrm{NS}^{\mathrm{a}}$ \\
\hline FEV1/FVC (after BD) & $65.91 \pm 6.73$ & $71.77 \pm 13.02$ & $0.025^{*_{a}}$
\end{tabular}

a: Independent t test, b: Mann Whitney test, c: Chi- square, data are represented as mean \pm SD or median (interquartile range), A. fumigatus: Aspergillus fumigatus, BD: bronchodilator, FEV1\%: forced expiratory volume in first second, FEV1/FVC\%: forced expiratory volume in first second: forced vital capacity, \% pred: percentage of predicted value, $P>0.05$ is not significant (NS). 


\section{Discussion}

This study was conducted on 60 individuals; 30 were diagnosed as bronchial asthma (according to GINA 2018) ${ }^{12}$, and 30 were diagnosed as ACO according to sin et al. ${ }^{4}$ criteria proposed in 2016.

All ACO patients encompassed in our study were smokers, 13 of them were active smokers with median smoking index 25 , and the remaining were passive smokers for more than 10 years. This was significantly higher than in asthmatic group which comprised only 1 active and 1 passive smokers. This finding agrees with Park et al. ${ }^{15}$ and Sevimli et al. ${ }^{16}$ who found a significantly higher percentage of smokers in ACO group versus the asthmatic patients. ACO criteria mandated being smoker for 10 pack/year or exposed to equivalent indoor or outdoor air pollution. ${ }^{4}$

Both groups were comparable regarding the presence of atopy (associated allergic rhinitis and lor chronic urticaria). On the contrary, Sevimli et al. ${ }^{16}$ evaluated the presence of allergic comorbidity in ACO patients and found that it was significantly lower than that in patients with asthma $(P<0.05)$. As stated in GINA $2020^{3}$, ACO patient has many phenotypes one of them is an adult with long history of asthma whose pulmonary functions show persistent airflow limitation. So, this may explain why both groups were comparable in our study regarding atopic status.

Blood eosinophil count and serum total IgE level results showed significantly higher levels amongst ACO patients compared to asthma patients ( $P=0.003,0.007$ respectively). Results for serum total IgE were in line with that of Papi et al. ${ }^{17}$ and Lee et al. ${ }^{18}$ who found significantly higher serum total IgE levels among ACO group versus asthma patients. However, unlike our study, Lee et al. ${ }^{18}$ found that blood eosinophil count was lower among ACO group compared to asthma patients $(P=0.02)$. On the other hand, Park et al. ${ }^{15}$ and Sevimli et al. ${ }^{16}$ found no significant difference in serum levels of total IgE or blood eosinophil count between asthmatics and ACO patients. This discrepancy could have many possible explanations. First, it could be due to the high prevalence of associated atopy among ACO patients in our study (66.7\%). In addition, blood eosinophil count above $300 / \mu \mathrm{L}$ was a minor criterion for diagnosing ACO patients according to Sin et al., 2016. Lastly, all ACO patients in our study were either active or passive smokers in contrast to the asthma only group which included only 2 smokers. Smoking is known to cause elevation of eosinophil count in blood. ${ }^{19}$

Pulmonary function tests were done for both groups in our study and was a cornerstone for detecting ACO patients. The $\mathrm{FEV}_{1} / \mathrm{FVC} \%$ ratio didn't exceed $70 \%$ in ACO patients satisfying one of the major criteria for diagnosis. ACO group showed lower post BD FEV 1 and $\mathrm{FEV}_{1} / \mathrm{FVC}$ $\%$ than that of asthma group. These results are consistent with previous research. ${ }^{15,20,21}$

Many studies stated that the diagnosis of fungal sensitization can be made either with by skin testing or measuring specific IgE levels. ${ }^{8,22}$ Accordingly, we used both methods which yielded almost similar results with concordance of $85.7 \%$ between both techniques. Our results showed that IgE sensitization to A. fumigatus (by skin test or slgE) was found in $26.7 \%$ of asthma patients. This corroborates previous results such as $21.5 \%{ }^{23}, 27.6 \%^{24}$, and $35.1 \%^{25}$. In contrast, another study found that only $6.6 \%$ of asthmatic patients were sensitized to $A$. fumigatus. ${ }^{2}$ The mean age of asthmatic patients included in their study was $16.61 \pm 12.31$ years versus $47.63 \pm 7.42$ years in our asthma group which may explain the discrepancy in results. On the other hand, another study reported that $39.5 \%$ of asthmatic patients were Aspergillus sensitized. ${ }^{27}$ This discrepancy could be attributed to varieties in genetics, race, geographical and climatic conditions.

Our results revealed that that $43.3 \%$ of ACO patients were sensitized to $A$. fumigatus. On the contrary, Tanosaki et al. ${ }^{28}$ mentioned that fungal sensitization collectively (Aspergillus, Alternaria, cladosporium, Penicillium and Trichophyton) in ACO patients was only $29 \%$. Diagnosis was only done by detecting serum specific IgE to these allergens without performing SPT. This may explain the higher prevalence in our study.

Prevalence of sensitization to $A$. fumigatus was higher in ACO patients (43.3\%) compared to asthmatic patients (26.7\%), but the difference between both groups was not significant $(P=0.17)$. Prior studies have noted that sensitization to $A$. fumigatus was prevalent 
among COPD patients $13 \%{ }^{11}, 18 \%^{29}$. This considerable prevalence among pure COPD patients may explain why ACO patients show slightly higher results than asthma in our study.

In our study we compared $A$. fumigatus IgEsensitized and non-sensitized asthmatic patients as regards the various parameters in our study. No significant difference was revealed regarding age and disease duration. Concerning pulmonary functions, sensitized asthmatic patients showed significantly lower post-BD FEV $1 \%$ and FEV1/FVC $(P=0.001$ and 0.002 respectively) compared to non-sensitized patients. These results suggest that fungal sensitization could be an important risk factor for asthma severity. This comes in line with previous studies observations, where poorer lung functions were noted among Aspergillus sensitized patients with asthma compared to non-sensitized ones. ${ }^{30,31}$ Another important finding was that higher total IgE and blood eosinophil count was observed among sensitized group; nevertheless, unlike previous studies $^{30}$, it did not reach statistical significance ( $P$ value $0.55,0.07$ respectively). This might have happened due to the small sample size of sensitized asthmatic patients in our study.

On comparing aspergillus sensitized and nonsensitized ACO patients, results were comparable regarding pulmonary functions, total IgE and smoking status. Only blood eosinophil count was significantly higher among sensitized compared to non-sensitized ACO patients $(P=0.017)$. On the contrary, Tanosaki et $a^{28}{ }^{28}$ compared fungal sensitized with nonsensitized ACO patients and found that total IgE was significantly higher among sensitized patients $(p=0.003)$, while there was no difference in blood eosinophil counts between both groups. In addition, airflow limitation was comparable between both groups $(P=0.139)$. Tanosaki et al. studied fungal sensitization globally, while our study was on $A$. fumigatus only, this may explain inconsistency of results.

On comparing all study patients $A$. fumigatus sensitized to non-sensitized ones, it was obvious that ACO patients constituted higher proportion in sensitized group (62\%) than in non-sensitized group (43.5\%), also Blood eosinophil count was significantly higher among $A$. fumigatus sensitized patients $(P=0.002)$. This corroborates a recent study conducted in $2020^{32}$. Besides,
post-BD $\mathrm{FEV}_{1} / \mathrm{FVC}$ of patients sensitized to aspergillus was significantly lower than in nonsensitized group $(P=0.025)$. Otherwise, no significant difference was found as regards age, disease duration, total IgE and post-BD FEV1\%.

Our data suggest that sensitization to $A$. fumigatus may play a role in ACO disease course. Further studies on ACO patients with larger sample size should be conducted to help understand the course of the disease and best ways for management. Also, screening all ACO patients for fungal sensitization might be beneficial to ameliorate its lines of management.

\section{Author Contributions}

MAE designed the idea and the tools of the study. NO revised step by step the recruitment of patients, and the tools used, $\mathrm{MH}$ collected, analyzed, and interpreted the data., AM made the statistical analysis and interpreted the results. NS proposed the methodology of the study, and ST wrote the manuscript. All authors agreed with the results and conclusions of this article. All authors read and approved the final manuscript.

\section{Declaration of Conflicting Interests}

The author(s) declared no potential conflicts of interest with respect to the research, authorship, and/or publication of this article.

\section{Funding}

This research did not receive any specific grant from funding agencies in the public, commercial, or notfor-profit sectors.

\section{Ethical approval}

Ethical approval was obtained by the Research Ethics Committee of faculty of medicine, Ain Shams University (FMASU REC).

\section{Informed consent}

Informed consent was obtained from all individual participants included in the study.

\section{References}

1. Cosío BG, Dacal D, Pérez de Llano L. (2018) Asthma-COPD overlap: identification and optimal treatment. Ther Adv Respir Dis.; 12:17534666 18805662. doi:10.1177/1753466618805662 
2. Miravitlles $M$, Soler-Cataluña JJ, Calle $M$, et al. (2017) Spanish Guidelines for Management of Chronic Obstructive Pulmonary Disease (GesEPOC) 2017. Pharmacological Treatment of Stable Phase. Guía española de la enfermedad pulmonar obstructiva crónica (GesEPOC) 2017. Tratamiento farmacológico en fase estable. Arch Bronconeumol.; 53(6):324-335. doi:10.1016/j.arbres.2017.03.018

3. GINA 2020 Global Initiative for Asthma (GINA)Global Strategy for Asthma Management and Prevention, 2008. Updated 2018 (2020) Available at: https://ginasthma.org/wp-content/ uploads/2020/06/GINA-2020-report_20_06_041-wms.pdf, last accessed 28th May 2020.

4. Sin DD, Miravitlles M, Mannino DM, et al. (2016) What is asthma-COPD overlap syndrome? Towards a consensus definition from a round table discussion. Eur Respir J. 48(3):664-673. doi:10.1183/13993003.00436-2016

5. Leung JM, Sin DD. (2017) Asthma-COPD overlap syndrome: pathogenesis, clinical features, and therapeutic targets. BMJ.; 358:j3772. doi:10.1136/bmj.j3772

6. Hosseini M, Almasi-Hashiani A, Sepidarkish M, et al. (2019) Global prevalence of asthma-COPD overlap (ACO) in the general population: a systematic review and meta-analysis. Respir Res.; 20(1):229. doi:10.1186/s12931-019-1198-4

7. Kennedy JL, Heymann PW, Platts-Mills TA. (2012) The role of allergy in severe asthma. Clin Exp Allergy.; 42(5):659-669. doi:10.1111/j.13652222.2011.03944.x

8. Agarwal R, Gupta D. (2011) Severe asthma and fungi: current evidence. Med Mycol. 49 Suppl 1:S150-S157. doi:10.3109/13693786.2010.50475 2

9. Denning DW, Pashley C, Hartl D, et al. (2014) Fungal allergy in asthma-state of the art and research needs. Clin Transl Allergy.; 4:14. doi:10.1186/2045-7022-4-14

10. O'Driscoll BR, Powell G, Chew F, et al. (2009) Comparison of skin prick tests with specific serum immunoglobulin $E$ in the diagnosis of fungal sensitization in patients with severe asthma. Clin Exp Allergy; S39(11):1677-1683. doi:10.1111/ j.1365-2222.2009.03339.x

11. Bafadhel M, McKenna S, Agbetile J, et al. (2014) Aspergillus fumigatus during stable state and exacerbations of COPD. Eur Respir J.; 43(1):64-71. doi:10.1183/09031936.00162912

12. Global Initiative for Asthma (GINA) (2008) Global Strategy for Asthma Management and Prevention. Updated 2018 (2018) Available at:https://ginasthma.org/wp-content/uploads/
2018/04/wms-GINA-2018-report-tracked_v1.3. pdf, last accessed Novemeber, 2018.

13. Miller MR, Hankinson J, Brusasco V, et al. (2005) Standardisation of spirometry. Eur Respir J.; 26(2):319-338. doi:10.1183/09031936.05.00034 805

14. Arbes SJ Jr, Gergen PJ, Elliott L, et al. (2005) Prevalences of positive skin test responses to 10 common allergens in the US population: results from the third National Health and Nutrition Examination Survey. J Allergy Clin Immunol. 116(2):377-383. doi:10.1016/j.jaci.2005.05.017

15. Park SY, Jung H, Kim JH, et al. (2019) Longitudinal analysis to better characterize Asthma-COPD overlap syndrome: Findings from an adult asthma cohort in Korea (COREA). Clin Exp Allergy; 49(5):603-614. doi:10.1111/cea.13339

16. Sevimli N, Yapar D, Türktaş H. (2019) The Prevalence of Asthma-COPD Overlap (ACO) Among Patients with Asthma. Turk Thorac J.; 20(2):97-102. doi:10.5152/TurkThoracJ.2018.180 55

17. Papi A, Romagnoli M, Baraldo S, et al. (2000) Partial reversibility of airflow limitation and increased exhaled NO and sputum eosinophilia in chronic obstructive pulmonary disease. Am J Respir Crit Care Med.; 162(5):1773-1777. doi:10.1164/ajrccm.162.5.9910112

18. Lee HY, Kang JY, Yoon HK, et al. (2014) Clinical characteristics of asthma combined with COPD feature. Yonsei Med J. 55(4):980-986. doi:10. 3349/ymj.2014.55.4.980

19. Higuchi T, Omata F, Tsuchihashi K, el al. (2016) Current cigarette smoking is a reversible cause of elevated white blood cell count: Cross-sectional and longitudinal studies. Prev Med Rep.; 4:417-422.doi:10.1016/j.pmedr.2016.08.009

20. De Marco R, Marcon A, Rossi A, et al. (2015)NAsthma, COPD and overlap syndrome: a longitudinal study in young European adults. Eur Respir J.; 46(3):671-679. doi:10.1183/09031936.00008615

21. Duong-Quy S, Tran Van H, Vo Thi Kim A, et al. (2018) Clinical and Functional Characteristics of Subjects with Asthma, COPD, and Asthma-COPD Overlap: A Multicentre Study in Vietnam. Can Respir J. 2018:1732946. doi:10.1155/2018/ 1732946

22. Kwizera R, Musaazi J, Meya DB, et al. (2019) Burden of fungal asthma in Africa: A systematic review and meta-analysis. PLoS One.; 14(5):e0216568. doi:10.1371/journal.pone.0216568

23. Malo JL, Paquin R. (1979) Incidence of immediate sensitivity to Aspergillus fumigatus in a North 
American asthmatic population. Clin Allergy.; $9(4): 377-384$. doi:10.1111/j.13652222.1979.tb02496.x

24. Maurya V, Gugnani HC, Sarma PU, et al. (2005) Sensitization to Aspergillus antigens and occurrence of allergic bronchopulmonary aspergillosis in patients with asthma. Chest; 127(4):1252-1259. doi:10.1378/chest.127.4.1252

25. Byeon JH, Ri S, Amarsaikhan O, et al. (2017) Association Between Sensitization to Mold and Impaired Pulmonary Function in Children With Asthma. Allergy Asthma Immunol Res.; 9(6):509-516. doi:10.4168/aair.2017.9.6.509

26. Ishak SR, Abd El Sayed STK, Wahba NS. (2020) Prevalence of common sensitizing aeroallergens in Egyptian asthmatic patients. World Allergy Organ J. Apr 17; 13(4):100115. doi: 10.1016/j.waojou.2020.100115.

27. Agarwal R, Gupta D, Aggarwal AN, et al. (2006) Allergic bronchopulmonary aspergillosis: lessons from 126 patients attending a chest clinic in north India. Chest.; 130(2):442-448. doi:10.1378/ chest.130.2.442
28. Tanosaki T, Fukunaga K, Miyazaki M, et al. (2016) Clinical Characteristics of Asthma--COPD Overlap Syndrome Patients with Fungal Sensitization. Am J Respir Crit Care; 193: A6245.

29. Everaerts S, Lagrou K, Dubbeldam A, et al. (2017) Sensitization to Aspergillus fumigatus as a risk factor for bronchiectasis in COPD. Int J Chron Obstruct Pulmon Dis. Aug 31;12:2629-2638. doi: 10.2147/COPD.S141695.

30. Fairs A, Agbetile J, Hargadon B, et al. (2010) IgE sensitization to Aspergillus fumigatus is associated with reduced lung function in asthma. Am J Respir Crit Care Med.; 182(11):1362-1368. doi:10.1164/rccm.201001-00870C

31. Mansour AE, El-Desouky ME, Bedeer T. (2012) Effect of Aspergillus fumigatus sensitization and colonization on lung function and airways inflammation in asthma. Egyptian Journal of Chest Diseases and Tuberculosis, 61. 243-246. doi:10.1016/j.ejcdt.2012.09.006

32. Kono Y, To M, Tsuzuki R. et al. (2020) Pulmonary emphysema is associated with fungal sensitization in asthma. J Thorac Dis. Oct; 12(10):5879-5886. doi: 10.21037/jtd-20-995. 